\title{
O efeito do exercício de estimulação da memória em idosos saudáveis
}

\author{
THE EFFECT OF MEMORY STIMULATION PRACTICES AS A THERAPEUTIC \\ METHOD ON HEALTHY ELDERS
}

\author{
EL EFECTO DEL EJERCÍCIO DE ESTIMULACIÓN DE LA MEMORIA \\ COMO MÉTODO TERAPÉUTICO EN ANCIANOS SALUDABLES
}

\author{
Juliana Nery de Souza ${ }^{1}$, Eliane Corrêa Chaves ${ }^{2}$
}

\begin{abstract}
RESUMO
Considerando a freqüente queixa dos idosos frente ao seu desempenho mnemônico, este estudo tem por objetivo investigar o efeito da estimulação da memória sobre o desempenho no MiniExame do Estado Mental (MEM), e observar correlação sóciodemográfica com o desempenho cognitivo. O MEM foi aplicado em 46 idosos integrantes de uma oficina de memória, sem diagnóstico médico de demência, antes e após a execução das atividades da mesma. Observou-se correlação e significância estatística entre os escores dos MEMs aplicados antes e após a oficina, porém não houve associação significante estatisticamente entre esse desempeho e as variáveis sócio-demográficas.
\end{abstract}

\section{DESCRITORES}

Idoso.

Memória.

Cognição.

\begin{abstract}
Considering the constant complaints of the elderly regarding their memory performance, this study aims to investigate the effect of memory stimulation on the performance in the Mini Mental State Examination (MMSE) and to observe the socio-demographic correlation with cognitive performance. The MMSE was applied in 46 elders without dementia diagnosis that participated in a memory workshop before and after the activities of the workshop were carried out. Correlation and statistical significance was observed between the MMSE's scores applied before and after the workshop, but there were no statistically significant association between this performance and the socio-demographic variables.
\end{abstract}

\section{KEY WORDS}

Aged.

Memory.

Cognition.

\section{RESUMEN}

Considerando las frecuentes quejas de los ancianos en relación a su desempeño mnemónico, este estudio tiene por objetivo investigar el efecto de la estimulación de la memoria relacionada a su desempeño, en el Mini Mental State Examination (MMSE) y observar la correlación sociodemográfica con el desempeño cognitivo. El MMSE fue aplicado en 46 ancianos integrantes de un taller de memoria, sin diagnóstico médico de demencia, antes y después de realizar las actividades del mismo. Fue observado correlación y significancia estadística entre los scores del MMSE aplicados antes y después del taller, no siendo observado asociación significativa estadísticamente con las variables sociodemográficas.

\section{DESCRIPTORES}

Anciano.

Memoria.

Cognición.
1 Aluna do 40 ano do curso de Graduação de Enfermagem da Escola de Enfermagem da USP. Bolsista CNPq. juli3@bol.com.br

2 Enfermeira. Professora Doutora do Departamento de Enfermagem MédicoCirúrgica da EEUSP. eccsp@yahoo.com 
Juliana Nery de Souza Eliane Corrêa Chaves

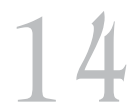

Rev Esc Enferm USP 2005; 39(1): 13-9.

\section{INTRODUÇÃO}

O crescimento da população mundial idosa é um fenômeno que vem ocorrendo nas últimas décadas com mais intensidade nos países em desenvolvimento. O Brasil tem aproximadamente 15 milhões de pessoas com 60 anos ou mais $(8,6 \%$ da população brasileira). Nos próximos 20 anos a população idosa do Brasil poderá ultrapassar os 30 milhões de pessoas e deverá representar quase $13 \%$ da população ao final deste período, no mundo, em 2050, um quinto da população será de idosos $^{(1)}$. As atuais conquistas médico-tecnológicas da medicina moderna têm possibilitado a prevenção e cura de doenças que antes eram consideradas fatais, o que reduziu a mortalidade, aumentou a expectativa de vida e, conseqüentemente, elevou a taxa da população idosa, mesmo em países em desenvolvimento, onde o acesso aos serviços médicos é restrito.

Frente a esses dados, levanta-se uma preocupação inquietante com relação às afecções e distúrbios advindos da idade, principalmente os de origem crônico-degenerativos, e sua repercussão no processo saúde-doença desta população.

Dentre as várias alterações fisiológicas decorrentes do processo de envelhecimento as funções do sistema nervoso central, principalmente as de origem neuropsicológicas envolvidas no processo cognitivo, tais como o aprendizado e memória, constituem um dos principais alvos de pesquisas realizadas sobre senescência ${ }^{(2)}$, já que estas alterações podem comprometer o bem estar bio-psico-social do idoso impedindo a continuidade da sua vida social de forma participativa, interagindo com os familiares em particular e com a sociedade no geral.

Tomados pela concepção do processo de envelhecimento como um acontecimento patológico e marco inicial para a contagem regressiva de suas vidas, alguns idosos abandonam a preocupação de se manterem participativos no ambiente social em que vivem, entregando-se à aposentadoria de maneira passiva, inativa e cada vez menos reflexiva, o que, por sua vez, pode representar um prejuízo incondicional à saúde mental e física do indivíduo ${ }^{(3-4)}$, além de constituir-se fator de risco para o declínio cognitivo e para a demência ${ }^{(5)}$.

Estabelece-se um ciclo vicioso, no qual a degeneração psicobiológica própria do envelhecimento induz a inatividade e a pouca requisição de processos cognitivos, que por sua vez acelera a degeneração psicobiológica e assim por diante.
Embora a aposentadoria possa apresentar caráter prejudicial para alguns idosos, seu impacto varia de acordo com características individuais ${ }^{(6)}$, podendo se constituir um desafio que, a partir de processos adaptativos, pode ser superado pela maioria dos indivíudos ${ }^{(7)}$.

Alguns estudos sugerem a existência de fatores relacionados à saúde, eventos biológicos $\mathrm{e}$ ao modo de vida do indivíduo como possíveis determinantes da taxa de envelhecimento $\operatorname{cognitivo}^{(8)}$.

Considerável parte da população idosa queixa-se da dificuldade de armazenar informações e de resgatá-las (não se lembram de nomes de pessoas conhecidas, de compromissos importantes, como tomar remédio; não se lembram onde deixaram certos objetos pessoais, esquecem-se do fogo aceso, do ferro ligado, entre inúmeros outros exemplos que prejudicam seu desempenho e que podem por em risco sua saúde e segurança, afetando negativamente seu cotidiano), além de referirem seu prejuízo ocupacional e social diante dessas alterações decorrentes da velhice, levando muitos ao auto-abandono, perda da autoestima e seu isolamento da sociedade e até mesmo do ambiente familiar. No entanto, alguns idosos conseguem recorrer a várias estratégias para lidar com esses eventos de vida estressantes e com as demandas do dia-a dia $^{(9)}$, utilizando recursos pessoais (físicos, psicológicos e de competência e ambientais (recursos materiais e suporte social e familiar) ${ }^{(10)}$.

Levando-se em consideração essas queixas que os idosos freqüentemente apresentam e o desconhecimento a respeito da fronteira entre a normalidade neurobiológica, neuropsicológica e comportamental do idoso e a patologia como a demência do tipo Alzheimer, existe a preocupação de eventos considerados fisiológicos no processo de envelhecer evoluírem para um transtorno severo de declínio cognitivo.

Dados epidemiológicos sugerem que o comprometimento da memória associado ao envelhecimento (AAMI - age-associated memory impairment) é mais um fenômeno correlato ao envelhecimento do que um sinal de evolução da normalidade para o estado patológico como as demências, sugerindo que diagnósticos mais confiáveis (do que somente AAMI) são necessários para se usarem em estudos que estão tentando identificar fatores de risco para demência ou encontrar um tratamento precoce para este tipo de transtorno cognitivo ${ }^{(11)}$. Não há estudos conclusivos, no entanto, que afirmem categori- 
camente que o comprometimento da memória no idoso se trata de acontecimento inexorável do processo de envelhecimento ou se é resultado de um fenômeno puramente natural ou multifatorial, o qual resulta entre outras coisas do decréscimo de estímulos sociais, psicológicos e biológicos.

Independente desta importante e relevante preocupação com a possível evolução do declínio cognitivo fisiológico para a demência, as queixas subjetivas que os idosos apresentam a respeito de seu desempenho mnemônico são reais e devem ser acolhidas dentro da compreensão de que sendo um processo natural ou não comprometem seu cotidiano, sua auto-estima e relacionamento social e que necessitam de intervenções que amenizem ou até eliminem este transtorno, principalmente porque, embora o esquecimento se constitua uma das principais queixas entre os idosos, não se pode esquecer que outras funções cognitivas como a capacidade de leitura, o conhecimento dos significados das palavras e a utilização dos mesmos, permanecem inalterados ou pouco comprometidos com o envelhecimento ${ }^{(12)}$, devendo portanto, serem preservados e valorizados no processo de envelhecer.

Exemplifica-se uma teoria $^{(13)}$ não descrita na literatura científica que relaciona "perda" de memória não somente às alterações anátomo-fisiológicas, mas também à diminuição do exercício cognitivo e da motivação no processo de aprendizagem e memória, baseado na idéia de que para garantir que uma certa informação seja preservada é preciso que ela seja solicitada, ou seja, para funcionar de forma adequada, o cérebro precisa ser solicitado. Assim, como se recomenda exercícios físicos aos idosos para manter sua saúde física, deve-se estimular o cérebro com atividades que exijam atenção, concentração e pensamento lógico, o que contribui para o aumento da densidade sináptica cerebral, cuja rede de transmissão é responsável pela dinâmica e plasticidade do cérebro.

Isso talvez explique o excelente desempenho cognitivo e intelectual apresentados por muitos idosos ativos que apesar da aposentadoria não deixaram de aprimorar seus conhecimentos, através da leitura, exercício do raciocínio e da lógica e aquisição de novas informações.

Frente a esses dados, postula-se a aplicação do exercício da memória, através da sua estimulação/motivação, como método terapêutico na recuperação e/ou manutenção dessa função neural em idosos cujo processo físiológico do envelhecimento é tido como natural, ou seja, sem comprometimentos neurológicos pré-existentes.

\section{OBJETIVOS}

Esse estudo tem como objetivos verificar a existência da relação entre o desempenho mnemônico e a constante estimulação dessa função neural; e verificar a presença de correlação de aspectos sócio-demográficos (idade, escolaridade, presença de companheiro, inserção em atividades sociais, físico-esportivas, culturais e de lazer) e aspectos neuropsicológicos (estado de humor - ansiedade e depressão, e queixas mnemônicas dos idosos) com o desempenho das funções cognitivas.

\section{METODOLOGIA}

O estudo foi realizado em São Paulo, na Escola de Enfermagem da USP, e os dados foram coletados somente após análise e aprovação do Comitê de Ética dessa mesma escola.

Para o alcance dos objetivos propostos aplicou-se o Mini-Exame do Estado Mental (MEM) ${ }^{(14)}$, traduzido e validado para a população brasileira ${ }^{(15)}$, em 46 pessoas com mais de 60 anos, sem diagnóstico médico de demência ou de qualquer outra afecção cognitiva ou neurológica, além de não estarem recebendo medicação psicoativa, concordarem em participar do estudo e serem freqüentadores de uma oficina de memória organizada pelas pesquisadoras.

Inicialmente todos os participantes preencheram um questionário de caracterização sóciodemográfica composto por questões relativas a dados gerais de caracterização pessoal.

O MEM foi aplicado antes do início das atividades da oficina e ao final dessa, com o intuito de comparar os escores, bem como, de verificar aumento dos mesmos após as atividades estimuladoras. Essas atividades ocorreram em 8 reuniões, com duração de 2 horas cada, totalizando 16 horas de atividades. Elas consistiram em apresentação de palestras, aplicação de jogos, atividades e exercícios que exercitassem e estimulassem o raciocínio, os diferentes tipos de memória e as demais funções cognitivas. Esses exercícios eram essencialmente compostos por resolução de problemas, cálculos, memorização visual, leitura entre outros.

Além do MEM, foram aplicados aos idosos, o Inventário de Ansiedade Traço-Estado de Spilberger - IDATE ${ }^{(16)}$ e o Inventário de Depressão de Beck ${ }^{(17)}$, ambos com o intuito de verificar a presença de transtornos de humor que pudessem interferir nos resultados de avaliação cognitiva.
O efeito do exercício de estimulação da memória em idosos saudáveis 
Juliana Nery de Souza Eliane Corrêa Chaves

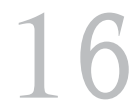

Rev Esc Enferm USP 2005; 39(1): 13-9.
O MEM é composto por diversas questões tipicamente agrupadas em sete categorias, cada uma delas planejada com o objetivo de avaliar componentes da função cognitiva, como orientação têmporo-espacial (5 pontos cada), retenção ou registro de dados (3 pontos), atenção e cálculo (5 pontos), memória ( 3 pontos), linguagem ( 8 pontos) e capacidade construtiva visual (1 ponto). O escore do MEM pode variar de um mínimo de 0 até um total máximo de 30 pontos. A escala é simples de usar e pode ser facilmente administrada em 5-10 minutos. Possui pontuação de corte de 24 pontos, na qual escores abaixo deste valor são indicadores para o diagnóstico de demência ${ }^{(18)}$, embora, alguns autores ${ }^{(15,19)}$ sugiram pontos de cortes diferenciados, dependendo do grau de escoridade, este estudo não se ateve à esta questão, considerando que o objetivo do mesmo não era de diagnosticar possíveis quadros demenciais, mas de observar melhora no dempenho cognitivo após atividades estimuladoras de uma oficina de memória sugerindo.

Quanto ao preenchimento do MEM, as respostas foram obtidas mediante perguntas de avaliação clínica dirigidas, cujas respostas são categorizadas em escores com base no julgamento da pessoa que está dirigindo a avaliação e não do respondente, embora os escores estejam previamente estabelecidos no próprio instrumento.

O Inventário de Ansiedade Traço-Estado (IDATE) apresenta como característica principal a mensuração de aspectos inespecíficos envolvidos em situações problemáticas ou estressantes, como tensão, nervosismo, irritabilidade, apreensão, preocupação e outros. O IDATE foi projetado para ser auto-aplicável, e pode ser aplicado individualmente ou em grupos. É constituído por dois instrumentos independentes - traço de ansiedade e estado de ansiedade - os quais enfocam abordagens distintas de ansiedade. Visando o caráter emocional transitório do estado de ansiedade, neste estudo foi utilizada apenas a escala traço de ansiedade (o indivíduo responde como se sente habitualmente, sendo que o traço de ansiedade se refere a diferenças individuais relativamente estáveis em propensão à ansiedade, isto é, a diferenças na tendência de reagir a situações percebidas como ameaçadoras), pois o objetivo de sua inclusão como instrumento de coleta de dados, como mencionado anteriormente, foi verificar a presença de distúrbios ou transtornos ansiosos nos indivíduos que pudessem interferir no desempenho dos idosos no MEM. O intrumento é constituído por 20 afirmações e nelas constam instruções de preenchimento impressas no formulário do testes, o qual não possui limite de tempo para ser respondido. A pontuação varia de 20 a 80 pontos, os indivíduos respondem a cada item do IDATE avaliando-se a si próprios numa escala de quatro pontos (1. quase nunca; 2 . às vezes; 3 . freqüentemente; 4 . quase sempre - todos para a escala traço). A amplitude de variação possível para soma das pontuações é de 20 a 80 pontos, em que pontuações baixas indicam baixa ansiedade e as mais altas o inverso. Os autores da escala não estabeleceram pontuação de corte para a normalidade, mas será utilizada uma categorização proposta em literatu$\mathrm{ra}^{(20)}$, na qual a pontuação de 20 a 40 pontos indica baixa ansiedade, de 40 a 60 média, e de 60 a 80 alta ansiedade.

O Inventário de Depressão de Beck é um instrumento de rastreamento de depressão e, portanto, não possui pretensão diagnóstica, visa simplesmente indicar quantas e quais são as pessoas que mereceriam uma avaliação clínica para o diagnóstico de depressão maior. Consiste em 21 itens compreendendo sintomas e comportamentos da esfera cognitiva, vegetativa e do humor, para serem avaliados em escala com quatro graus de intensidade ou severidade ( 0 a 3 ) e as pontuações podem variar de 0 a 63 . Foram utilizados, para diferenciar os níveis de sintomatologia depressiva, os critérios adotados por Gorestein, Andrade $^{(17)}$, nos quais uma nota de corte com pontuação igual ou superior a 16 para detectar disforia, compreendida como dor excessiva, angústia, agonia, agitação e inquietação ${ }^{(21)}$, e igual ou superior a 21 para categorizar indivíduos com depressão.

Ressaltando a importância dos aspectos éticos envolvidos em qualquer pesquisa, para todos os participantes, foi esclarecida a não obrigatoriedade de participação, os objetivos do estudo, o sigilo de nomes, bem como, a apresentação do termo de consentimento, no qual os participantes puderam optar ou não pela sua inclusão no estudo, estando cientes de que a recusa não traria qualquer ônus a eles, nem a exclusão da oficina de memória.

\section{RESULTADOS}

O estudo foi constituído por 46 indivíduos, sendo que 36 eram do sexo feminino (78,4\%) e 10 do sexo masculino (21,6\%), idade média de 71,86 anos, variando de 60 a 85 anos, com regular nível de escolaridade, sendo que apenas $26,1 \%$ dos idosos possuíam menos de 8 anos de escolarida- 
de formal. Com relação aos dados sócio-demográficos, esta população mostrou-se bastante homogênea, com altas taxas percentuais de participação social $(56,5 \%)$, cultural $(76,0 \%)$, físico-esportiva $(69,0 \%)$ e lazer $(95,7 \%)$, evidenciando que se trata de um grupo aparentemente ativo e inserido socialmente e, portanto, com possibilidade considerável de manutenção e estímulo de suas funções mentais.

Comparando-se os resultados do MEM antes das atividades estimuladoras do sistema cognitivo com os resultados deste mesmo teste após as atividades de intervenção, observamos que houve acréscimo significante estatisticamente $(\mathrm{p}=0.042)$ entre os valores nos diferentes momentos da oficina, conforme apresentando na Tabela 1.

Tabela 1- Distribuição da população, segundo as medianas dos escores do MEM 1 e 2. (São Paulo, 2002)

\begin{tabular}{c|c|c|c}
\hline MEM & $\begin{array}{c}\text { Freqüência } \\
\text { (N) }\end{array}$ & $\begin{array}{c}\text { Desvio } \\
\text { Padrão }\end{array}$ & Mediana \\
\hline MEM 1 & 29 & 200492 & 27.5 \\
\hline MEM 2 & 29 & 154649 & 28.0 \\
\hline
\end{tabular}

$p=0.042$

Além disso, observou-se que dos sete idosos com escores iguais ou inferiores a 24 , três obtiveram melhora do desempenho na categoria "memória" do MEM 2; três abandonaram a oficina, talvez até pela dificuldade imposta pelo déficit cognitivo e um permaneceu estável, mostrando que embora para este indivíduo as atividades não tenham tido caráter de recuperação da função mnemônica, pelo menos sua manutenção foi alcançada. Com relação aos valores obtidos nesta categoria "memória" do MEM de todos os idosos e não somente daqueles que tiveram escores iguais ou inferiores a 24 , notamos que, embora não houvesse alteração significativa entre os dois momentos, nos quais foram aplicados o MEM ( $\mathrm{p}=0.1824)$, dos onze idosos que apresentaram valores, no primeiro momento da oficina, iguais aos níveis $0-1-2$, sete $(63,6 \%)$ mudaram para o nível 3 no segundo momento da mesma, sugerindo uma possível melhora no desempenho da memória nestes idosos.

Do total de idosos que se submeteram ao MEM nos dois momentos da oficina (MEM1 antes e MEM2 após), $66 \%$ apresentaram um aumento nos escores após as atividades de intervenção que compunham a oficina. Os indivíduos que não obtiveram aumento do desempenho no MEM2 (34\%), apresentaram ou uma manutenção dos escores desse instrumento, ou um decréscimo, sendo que nestes detectou-se alguns casos de estado depressivo associado.

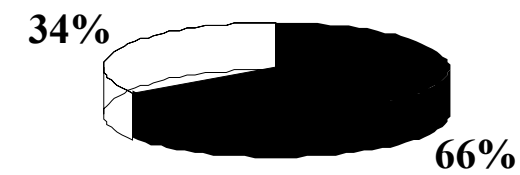

$\square$ idosos com aumento no MEM2
$\square$ idosos sem aumento no MEM2

Gráfico 1-Distribuição da população, segundo aumento dos escores no MEM2. (São Paulo, 2002)

$\mathrm{Na}$ tentativa de justificar as diferenças nos escores do MEM 2, buscamos verificar a existência de uma correlação entre esta desigualdade e a freqüência às atividades de intervenção, assim, aqueles que não possuíssem acréscimos no escore do MEM justificaríamos com uma menor freqüência nas atividades e, portanto, menor exposição aos exercícios estimuladores da memória. No entanto, observamos que tanto os idosos com freqüência maior que $75 \%$ quanto aqueles com freqüência menor que o mesmo valor, possuíam a mesma mediana de acréscimo nos escores do MEM 2, não alcançando, assim, associação significante entre esta variável e o decréscimo no MEM 2 ( $\mathrm{p}=0.8363$ ).

Mesmo não alcançando significância estatística entre a freqüência nas atividades e os resultados do MEM 2, podemos justificar o acréscimo obtido pela maioria dos idosos $(68,9 \%)$ às atividades de intervenção, uma vez que não sabemos qual o grau de envolvimento e aproveitamento de cada indivíduo nestas atividades, que não se limitavam apenas a exercícios aplicados durante cada sessão da oficina, mas também a práticas em casa e dicas de como solicitar as funções cognitivas no cotidiano. Assim, àqueles que não apresentaram qualquer progresso no escore do MEM 2, talvez tenha sido em virtude da diferença de aproveitamento e envolvimento nestas atividades. Além disso, não podemos descartar a hipótese deste acréscimo ter sido em função da própria familiaridade dos indivíduos com o teste (MEM), o que facilitaria uma melhora no desempenho.

Outro dado interessante, observado neste estudo e que contraria alguns autores ${ }^{(22-26)}$, foi a ausência de correlação significativa estatisticamente entre os resultados do MEM1 e o aumento dos escores no MEM2 e os aspectos sócio-demográficos (escolaridade, atividade profissional, física,
O efeito do exercício de estimulação da memória em idosos saudáveis 
Juliana Nery de Souza Eliane Corrêa Chaves sociais, físicas, culturais, lazer, quantidade de sono por dia, habito de leitura). Não se observou, nos idosos que possuem estas atividades, benefícios em relação ao desempenho cognitivo, embora não tenhamos avaliado a qualidade e quantidade destas atividades na vida diária destes indivíduos.

Observou-se também correlação negativa e significância estatística entre a idade e os resultados do MEM1, assim, nesta população quanto maiores as idades, menores foram os escores obtidos neste teste.

Os resultados do MEM aplicado no momento inicial do estudo foram comparados com os resultados dos inventários de ansiedade (IDATE) e depressão (BECK), e não se observou correlação e significância, mostrando que baixos escores não se justificam com estado depressivo ou ansioso. Além disso, observamos que dos sete idosos $(15,2 \%)$ que obtiveram escore igual ou inferior a 24 , somente um $(14,2 \%)$ apresentou quadro depressivo associado, detectado pelo inventário de Beck, que pudesse justificar seu baixo desempenho, dois $(28,5 \%)$ tiveram os resultados deste inventário anulados, pois tinham três ou mais questões do teste não respondidas. Ademais, notamos que destes sete idosos com escores iguais ou inferiores a 24 nenhum alcançou escore máximo de 3 pontos na categoria "memória" do MEM, mostrando que em todos eles este componente cognitivo apresentava um déficit.

Com relação às demais variáveis levantadas na caracterização da população (padrão de sono, acuidade visual e auditiva, hábito de leitura e autoavaliação do desempenho mnéstico - queixa de memória), não se observou correlação e significância estatística. As alterações de sono, acuidade visual e auditiva não foram, nesta população, fatores causais de baixos escores no MEM ou ausência de melhora nos escores desse exame, já que entre os idosos com altos escores, havia aqueles que mencionaram, durante o estudo, serem portadores de um ou mais dos déficits já mencionados, sem que isto alterasse seu desempenho cognitivo.

\section{CONCLUSÕES}

A partir da análise dos resultados deste estudo pôde-se observar que a maioria dos idosos apresentou aumento significante estatisticamente $(p=0.042)$ nos escores do MEM após a "Oficina de Memória", mostrando que houve uma melhora no desempenho cognitivo; no entanto, não podemos afirmar que este progresso tenha sido na função mnemônica, já que este teste avalia todas as funções cognitivas. Observamos que muitos dos idosos que tiveram seus escores aumentados, obtiveram também elevação na pontuação da categoria "memória" do MEM, mas nesta correlação não se obteve significância estatística. Por outro lado, não podemos descartar a hipótese de que este progresso no desempenho das funções cognitivas se deva à familiaridade com o teste, já que a freqüência nas atividades e, portanto, maior exposição a fatores estimulantes cognitivos, não se correlaciona com a diferença nos escores do MEM obtida nos dois momentos da oficina. Além disso, não sabemos qual o grau de envolvimento e participação de cada idoso nestas atividades.

Recomendamos que se continue avaliando mais precisamente o efeito das chamadas "oficinas de memória" no desempenho mnemônico, através de testes de avaliação mais precisos e específicos para memória, quantificando e avaliando o grau de envolvimento dos idosos nestas atividades, ao invés de se descartar esta possibilidade como método terapêutico e de importante valor para esta população em constante aumento mundialmente, considerando as implicações que estes déficits representam na qualidade de vida desse grupo de pessoas.

\section{REFERÊNCIAS}

(1) Fundação Instituto Brasileiro de Geografia e Estatística. Perfil dos idosos responsáveis pelos domicílios [online]. São Paulo; 2002. Disponível em: http://www.ibge.gov.br/home/estatistica/ populacao/perfilidoso/default.shtm $>$ (08 ago. 2002).
(2) Souza RR. Alterações anatômicas do sistema nervoso central associadas ao envelhecimento. In: Jacob Filho W, Carvalho Filho ET, editores. Envelhecimento do sistema nervoso e a dor no idoso monografias em geriatria III. São Paulo: FMUSP; 1996. 
(3) House JS, Landis KR, Umberson D. Social relationships and health. Science 1988; 241(4865):540-5.

(4) Abraham G, Kocher P, Goda G. Psychoanalysis and ageing. Intern Rev Psychoanal 1980; 7(2):147-55.

(5) Bahia VS, Caramelli P. Prevenção do declínio cognitivo relacionado à idade. In: Jacob Filho W, Carvalho ET, editores. Promoção da saúde do idoso. São Paulo: Lemos; 1998. p. 45-53

(6) Stoppe Jr A. Influência do processo de envelhecimento no quadro clínico de depressão em idosos. [dissertação] São Paulo (SP): Faculdade de Medicina da USP; 1995.

(7) Ferrari MAC. Lazer e ocupação do tempo livre. In: Papaléo Netto M, editor. Gerontologia: a velhice e o envelhecimento em visão globalizada. São Paulo: Atheneu; 1996. p. 98-105.

(8) Jolles J, Verhey FR, Riedel WJ, Houx PJ. Cognitive impairment in elderly people. Predisposing factors and implications for experimental drug studies. Drugs Aging 1995; 7(6):459-79.

(9) Goldstein LL. Stress e coping na vida adulta e na velhice. In: Neri AL, editora. Psicologia do envelhecimento: temas selecionados na perspectiva de curso de vida. São Paulo: Papirus; 1995. p. 145-58.

(10) Lazarus RS, Folkman S. Stress, appraisal and coping. New York: Springer; 1984.

(11) Hanninen T, Soininen H. Age-associated memory impairment. Normal aging or warning of dementia? Drugs Aging 1997; 11(6):480-9.

(12) Luders SLA, Storani MSB. Demência: impacto para a família e a sociedade. In: Papaléo Netto M, editor. Gerontologia: a velhice e o envelhecimento em visão globalizada. São Paulo: Atheneu; 1996. p. $146-59$.

(13) Lee Poncin M. Pense melhor, viva melhor: um guia prático de ginástica cerebral. Trad. Estela dos Santos Abreu. Rio de Janeiro: Salamandra; 1989.

(14) Folstein MF, Folstein SE, Mchugh PR. Mini-mental state: a practical method for grading the cognitive state of patients for the clinician. J Psychiatr Res 1975; 12(3):189-98.

(15) Bertolucci PHF, Brucki SMD, Campacci SR, Julianao Y. O miniexame do estado mental em uma população geral: impacto da escolaridade. Arq NeuroPsiquiatr 1994; 52:1-7.

(16) Spielberger CD, Gorsuch RL, Lushene RE. Inventário de Ansiedade Traço-Estado IDATE (State Anxiety Inventory - STAI). Rio de Janeiro: CEPA; 1979.
(17) Gorenstein C, Andrade L, editores. Escalas de avaliação clínica em psiquiatria e psicofarmacologia. São Paulo: Lemos; 2000. Inventário de Depressão de Beck - propriedades psicométricas da versão em português; p. 89-95.

(18) Tombaugh TN, Mcintyre NJ. The mini-mental state examination: a comprehensive review. J Am Geriatr Soc 1992; 40(9):922-35.

(19) Almeida OP. Miniexame do estado mental e o diagnóstico de demência. Arq Neuro-Psiquiatr 1998; 56:605-12.

(20) Chaves EC. Stress e trabalho do enfermeiro: a influência de características individuais no ajustamento e tolerância ao turno noturno. [tese] São Paulo (SP): Instituto de Psicologia da USP; 1994

(21) Dorland's illustrated medical dictionary. Philadelphia (PA): Sauders; 1994

(22) American College of Sports Medicine Position Stand. Exercise and physical activity for older adults. Med Sci Sports Exerc 1998; 30(6):992-1008.

(23) Christensen H, Machinnon A. The association between mental, social and physical activity and cognitive performance in young and old subjects. Age Ageing 1994; 23(5):435

(24) Yaffe K, Barnes D, Nevitt M, Lui LY, Covinsky K. A prospective study of physical activity and cognitive decline in elderly women: women who walk. Arch Intern Med 2001; 161(14):1703-8.

(25) Hassmen P, Koivula N. Mood, physical working capacity and cognitive performance in the elderly as related to physical activity. Aging (Milano) 1997; 9(1/2):136-42.

(26) Salemi G, Reggio A, Morgante L, Grigoletto F, Patti F, Meneghini F et al. Impact of sociodemographic characteristics on cognitive performance in $\mathrm{n}$ elderly Sicilian population. Neuroepidemiology 2002; 21(1):44-9.
O efeito do exercício de estimulação da memória em idosos saudáveis
Correspondência: Eliane Corrêa Chaves Av. Dr. Enéas de Carvalho Aguiar, 419 Cerqueira César - São Paulo CEP - 05403-000 - SP 